\title{
NOTE ON THE HURWITZ-NIELSEN REALISATION PROBLEM ${ }^{1}$
}

\author{
C. MACLACHLAN
}

\begin{abstract}
This problem, which is equivalent to the problem of showing that every finite subgroup of the Teichmüler modular group of a compact surface of genus $g$ has a fixed point in the corresponding Teichmüller space, is solved for $g=2$. The generalised problem for $n$-punctured surfaces of genus $g \geqslant 1$ and $1 \leqslant n \leqslant 4$ is also solved.
\end{abstract}

1. The Hurwitz-Nielsen realisation problem for compact surfaces $X_{g}$ of genus $g \geqslant 2$ may be stated as follows: Given a finite subgroup $G$ of the group of homotopy classes of self-homeomorphisms of $X_{g}$, can $X_{g}$ be endowed with a complex analytic structure $\alpha$ so that $G$ is realised as a group of conformal self-maps of the compact Riemann surface $\left(X_{g}, \alpha\right)$.

It is well known that this problem is equivalent to the Teichmüller fixed point problem i.e. every finite subgroup of the Teichmüller modular group of $X_{g}, \operatorname{Mod} X_{g}$, has a fixed point in the Teichmüller space of $X_{g}, T\left(X_{g}\right)$. This problem can be posed more generally for the Teichmüller modular group and the Teichmüller space of a finitely-generated Fuchsian group of the first kind and in this generality has been solved in the cases where the finite subgroup is soluble. (See [8], [4], [6], [9], [10].)

In this paper, detailed information on the structure of Mod $X_{2}$ is exploited to solve the Teichmüller fixed point problem in that case. In a similar vein, the cases corresponding to $n$-punctured surfaces of any genus $g \geqslant 1$ and $1 \leqslant n \leqslant 4$ are also solved. In the course of this latter proof, we obtain a new proof and an extension of a result on the torsion freeness of certain braid groups [3].

2. Let $\Gamma$ be a finitely-generated Fuchsian group of the first kind so that $\Gamma$ has a presentation of the form:

$$
\begin{gathered}
\text { Generators: } a_{1}, b_{1}, \ldots, a_{g}, b_{g}, e_{1}, e_{2}, \ldots, e_{r}, p_{1}, \ldots, p_{t}, \\
\text { Relations: } e_{i}^{m i}=1(i=1,2, \ldots, r), \prod_{j=1}^{g}\left[a_{j}, b_{j}\right] \prod_{k=1}^{r} e_{k} \prod_{l=1}^{t} p_{l}=1 .
\end{gathered}
$$

$\Gamma$ then has signature $\left(g ; m_{1}, \ldots, m_{r} ; t\right)$. We will use the special notation $\Gamma_{g, n}$ to denote a group of signature $(g ;-; n)$.

Received by the editors July 1, 1976 and, in revised form, September 1, 1976.

AMS (MOS) subject classifications (1970). Primary 32G15; Secondary 30A46.

Key words and phrases. Teichmüller space, Teichmüller modular group, Fuchsian group.

'Partial support by Carnegie Trust for Universities of Scotland. 
The Teichmüller modular group of $\Gamma, \operatorname{Mod} \Gamma$, is the quotient of $\mathfrak{A}(\Gamma)$, where $\mathfrak{U}(\Gamma)$ is the group of all type- and orientation-preserving automorphisms of $\Gamma$, by the inner automorphism of $\Gamma$. (See, for example, [6] for definitions and, in particular, for the isomorphism with the mapping-class group of the corresponding surface.) In particular, recall that $\operatorname{Mod} \Gamma_{g, t}$ is isomorphic to the mapping class group of the $t$-punctured surface of genus $g$. Let $T(\Gamma)$ denote the Teichmüller space of $\Gamma$.

3. Mod $\Gamma_{2,0}$ contains a normal subgroup of order 2 with quotient isomorphic to Mod $\Gamma$ where $\Gamma$ has signature $(0 ; 2,2,2,2,2,2 ; 0)$ [2], [6]. Now Mod $\Gamma$ is isomorphic to Mod $\Gamma_{0,6}$ (see, e.g., [6]). We first collect some facts that will be needed about $\operatorname{Mod} \Gamma_{0,6}$.

A presentation of Mod $\Gamma_{0,6}$ on five generators $\sigma_{1}, \sigma_{2}, \sigma_{3}, \sigma_{4}, \sigma_{5}$ is given in [7]. From this presentation the following are immediate:

(a) $\operatorname{Mod} \Gamma_{0,6} /\left[\operatorname{Mod} \Gamma_{0,6}, \operatorname{Mod} \Gamma_{0,6}\right]$ is cyclic of order 10,

(b) $\tau=\sigma_{2} \sigma_{3} \sigma_{4} \sigma_{5}$ has order 5 ,

(c) $\tau \notin\left[\operatorname{Mod} \Gamma_{0,6}, \operatorname{Mod} \Gamma_{0,6}\right]$.

In [6], it is proved that there are three elements of orders $4,5,6$, respectively, in Mod $\Gamma_{0,6}$, such that every element of finite order in Mod $\Gamma_{0,6}$ is conjugate to a power of one of them. Thus

(d) there are no elements of order 5 in $\left[\operatorname{Mod} \Gamma_{0,6}, \operatorname{Mod} \Gamma_{0,6}\right]$ by (b) and (c).

THEOREM 1. All finite subgroups of Mod $\Gamma_{2,0}$ are soluble.

Proof. Suppose, on the contrary, that $H \subseteq \operatorname{Mod} \Gamma_{2,0}$ is a finite nonsoluble subgroup. Let $\bar{H}$ denote its image in Mod $\Gamma_{0,6}$ so that $\bar{H}$ is also nonsoluble. By above, the only primes that can divide $O(\bar{H})$ are 2,3 and 5. Let $A=\bar{H} / \bar{H} \cap\left[\operatorname{Mod} \Gamma_{0,6}, \operatorname{Mod} \Gamma_{0,6}\right]$ so that $O(A) \mid 10$ by (a). By (d), only the

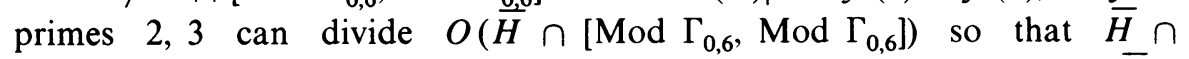
$\left[\operatorname{Mod} \Gamma_{0,6}, \operatorname{Mod} \Gamma_{0,6}\right]$ is soluble by Burnside's theorem. This implies $\bar{H}$ is soluble. This contradiction completes the proof.

From the results quoted in $\S 1$, follows

COROllary. Every finite subgroup of Mod $\Gamma_{2,0}$ has a fixed point in $T\left(\Gamma_{2,0}\right)$.

4. Before dealing with the Teichmüller fixed point problem for punctured surfaces, we prove an auxiliary result in greater generality than is immediately required, as its corollary may be of independent interest.

Consider $\Gamma_{g, n}$ where $n \geqslant 1, g \geqslant 1$. Recall that since each $\phi \in \mathfrak{U}\left(\Gamma_{g, n}\right)$ is type- and orientation-preserving, $\phi\left(p_{i}\right)=t_{i} p_{\phi(i)} t_{i}^{-1}$ where $i \mapsto \phi(i)$ is a permutation of the suffixes, $t_{i} \in \Gamma_{g, n}$ and $\Gamma_{g, n}$ has parabolic generators $p_{1}, p_{2}, \ldots, p_{n}$ as in (1). For $0 \leqslant t \leqslant n$, define

$$
\mathfrak{U}\left(\Gamma_{g, n}\right)_{t}=\left\{\phi \in \mathfrak{U}\left(\Gamma_{g, n}\right) \mid \phi\left(p_{i}\right)=t_{i} p_{i} t_{i}^{-1} \text { for } i=1,2, \ldots, t\right\}
$$

and $\left(\operatorname{Mod} \Gamma_{g, n}\right)_{t}$ its image in Mod $\Gamma_{g, n}$. Let $K$ be the normal closure in $\Gamma_{g, n}$ of $p_{t+1}, \ldots, p_{n}$ so that $\phi \in \mathfrak{U}\left(\Gamma_{g, n}\right)_{t}$ induces an automorphism of $\Gamma_{g, n} / K$. Provided that, when $g=1, t \geqslant 1, \Gamma_{g, n} / K$ is isomorphic to a Fuchsian group 
$\Gamma_{g, t}$ with signature $(g ;-; t)$. Thus there is an induced mapping

$$
\pi:\left(\operatorname{Mod} \Gamma_{g, n}\right)_{t} \rightarrow \operatorname{Mod} \Gamma_{g, v}
$$

THEOREM 2. The kernel of $\pi$ is torsion-free.

Proof. Let $\bar{\phi} \in\left(\operatorname{Mod} \Gamma_{g, n}\right)_{t}$ have finite order. Thus $\bar{\phi}$ can be realised conformally and there is an exact sequence

$$
1 \rightarrow \Gamma_{g, n} \stackrel{i}{\rightarrow} \Gamma \stackrel{\lambda}{\rightarrow}\langle\bar{\phi}\rangle \rightarrow 1
$$

where $\Gamma$ is a Fuchsian group. Without loss we can assume that $i$ is inclusion and that $\phi(\gamma)=\gamma_{2} \gamma \gamma_{2}^{-1}$ where $\lambda\left(\gamma_{2}\right)=\bar{\phi}[6]$. Since $\bar{\phi} \in\left(\operatorname{Mod} \Gamma_{g, n}\right)_{t}, K$ is normal in $\Gamma$, where $K$ is defined above.

Fill in the punctures of $U / \Gamma_{g, n}$ corresponding to the elements $p_{t+1}, \ldots, p_{n}$ to obtain a Riemann surface $S$ with distinguished points $Z_{t+1}, \ldots, Z_{n}$ such that $S-\left\{Z_{t+1}, \ldots, Z_{n}\right\}$ is conformally equivalent to $U / \Gamma_{g, n}$. Now let $\psi$ : $U \rightarrow S$ be the universal covering of $S$ and set $U_{0}=U-\psi^{-1}\left\{Z_{t+1}, \ldots, Z_{n}\right\}$. The conformal equivalence then lifts to give a conformal map $\sigma: U_{1} / K \rightarrow U_{0}$. Via $\sigma$, each element of $\Gamma$ acts on $U_{0}$ and so can be extended to $U$ since $U_{0}$ is $U$, the upper half-plane, with a countable discrete set of punctures. We thus obtain a mapping $\mu: \Gamma \rightarrow \Gamma^{\prime}$, a Fuchsian group, with kernel of $\mu=K$ and the image of $\Gamma_{g, n}$ isomorphic to $\Gamma_{g, t}$. Thus there is an exact sequence

$$
1 \rightarrow \Gamma_{g, t} \rightarrow \Gamma^{\prime} \rightarrow\langle\pi(\bar{\phi})\rangle \rightarrow 1,
$$

and so $O(\pi(\bar{\phi}))=O(\bar{\phi})$. Thus the kernel of $\pi$ is torsion-free.

Corollary. Let $B_{n}(M)$ and $F_{n}(M)$ denote the n-string and pure n-string braid groups of $M$, respectively (see, e.g., [1]). Then $B_{n}\left(X_{g}\right)$ for $g \geqslant$ $2, F_{n}\left(X_{g} \backslash\{m\right.$ points $\left.\}\right)$ for $g \geqslant 1, m \geqslant 1$ are torsion-free.

Proof. In the case $t=0$, the Nielsen isomorphism identifies Mod $\Gamma_{g, n}$ and $\operatorname{Mod} \Gamma_{g, 0}$ with the mapping-class groups of the $n$-punctured surfaces of genus $g \geqslant 2$ and the compact surfaces of genus $g \geqslant 2$, respectively. Using the results of Birman [1], the kernel of $\pi$ is then isomorphic to $B_{n}\left(X_{g}\right), g \geqslant 2$. From the same results one has that the pure mapping-class groups of $X_{\mathbf{g}} \backslash\{n$ points $\}$ and $X_{g} \backslash\{t$ points $\}, t \geqslant 1, g \geqslant 1$, are isomorphic to subgroups of $\left(\operatorname{Mod} \Gamma_{g, n}\right)_{t}$ and $\operatorname{Mod} \Gamma_{g, t}$, respectively, and that the kernel of $\pi$ is then isomorphic to $F_{n-1}\left(X_{g} \backslash\{t\right.$ points $\left.\}\right)$.

The torsion-freeness of $B_{n}\left(X_{g}\right), g \geqslant 2$, was first proved in [3].

5. The Teichmüller fixed point problem for $n$-punctured surfaces where $1 \leqslant n \leqslant 4$ and $g \geqslant 1$ is now considered. The following result was proved in a slightly different context in [5, p. 310].

THEOREM 3. Every finite subgroup of Mod $\Gamma_{g, 1}$ is cyclic.

TheOREM 4. Every finite subgroup of Mod $\Gamma_{g, n}$ where $g \geqslant 1$ and $1 \leqslant n \leqslant 4$ is soluble. 
Proof. Let $H \subseteq \operatorname{Mod} \Gamma_{g, n}$ be finite. Now $\left(\operatorname{Mod} \Gamma_{g, n}\right)_{n}$ is a normal subgroup of $\operatorname{Mod} \Gamma_{g, n}$ with quotient isomorphic to the symmetric group on $n$ letters. Since

$$
H \cap\left(\operatorname{Mod} \Gamma_{g, n}\right)_{n} \subseteq H \cap\left(\operatorname{Mod} \Gamma_{g, n}\right)_{1}=H_{1},
$$

$H$ is soluble if and only if $H_{1}$ is soluble. But under the mapping $\pi$ : $\left(\operatorname{Mod} \Gamma_{g, n}\right)_{1} \rightarrow \operatorname{Mod} \Gamma_{g, 1}, H_{1}$ is mapped isomorphically onto a subgroup of Mod $\Gamma_{g, 1}$ by Theorem 2. Thus by Theorem 3, $H_{1}$ is soluble.

COROLlaRY. Every finite subgroup of Mod $\Gamma_{g, n}$ has a fixed point in $T\left(\Gamma_{g, n}\right)$ for $g \geqslant 1,1 \leqslant n \leqslant 4$.

\section{REFERENCES}

1. J. S. Birman, Mapping class groups and their relationship to braid groups, Comm. Pure Appl. Math. 22 (1969), 213-238. MR 39 \# 4840.

2. J.S. Birman and H.M. Hilden, On the mapping class groups of closed surfaces as covering spaces, Advances in the Theory of Riemann Surfaces (Proc. Conf., Stony Brook, N.Y., 1969), Ann. of Math. Studies, No. 66, Princeton Univ. Press, Princeton, N.J., 1971, pp. 81-115. MR 45 \#1169.

3. E. Fadell and L. Neuwirth, Configuration spaces, Math. Scand. 10 (1962), 111-118. MR 25 \#4537.

4. S. Kravetz, On the geometry of Teichmüller spaces and the structure of their modular groups, Ann. Acad. Sci. Fenn. Ser. A I, No. 278 (1959), 1-35. MR 26 \#6402.

5. C. Maclachlan, Modular groups and fiber spaces over Teichmüller spaces, Discontinuous Groups and Riemann Surfaces (Proc. Conf., Univ. Maryland, College Park, Md., 1973), Ann. of Math. Studies, No. 79, Princeton Univ. Press, Princeton, N. J., 1974, pp. 297-314. MR 50 \# 7590.

6. C. Maclachlan and W. J. Harvey, On mapping-class groups and Teichmüller spaces, Proc. London Math. Soc. (3) 30 (1975), 496-512. MR 51 \# 10614.

7. W. Magnus, Uber Automorphismen von Fundamentalgruppen Berandeter Flächen, Math. Ann. 109 (1934), 617-646.

8. J. Nielsen, Abbildungsklassen endlicher Ordnung, Acta Math. 75 (1943), 23-115. MR 7, 137.

9. W. Fenchel, Estensioni di gruppi discontinui e trasformazioni periodiche delle superficie, Atti Accad. Naz. Lincei Rend. Cl. Sci. Fis. Mat. Nat. (8) 5 (1948), 326-329. MR 10, 558.

10. Bemaerkninger om endelige grupper af afbildningsklasser (Remarks on finite groups of mapping classes), Mat. Tidsskr. B 1950 (1950), 90-95. (Danish) MR 12, 349.

Department of Mathematics, University of Aberdeen, Kings College, Aberdeen, SCOTLAND 